\title{
The combination effects of body acupuncture and auricular acupressure compared to sham acupuncture for body weight control: study protocol for a randomized controlled trial
}

\author{
Linda L. D. Zhong ${ }^{1,2}$, Wai Kun ${ }^{1,2}$, Tsz Fung Lam¹,2, Shi Ping Zhang ${ }^{1,2}$, Jun Jun Yang ${ }^{1}$, Tat Chi Ziea ${ }^{3}$, Bacon Ng ${ }^{3}$
} and Zhao Xiang Bian ${ }^{1,2^{*}}$

\begin{abstract}
Background: Obesity is an increasingly prevalent chronic condition that is associated with serious morbidity and mortality. Excess body weight is a risk factor contributing to diseases such as hypertension, heart disease, hypercholesterolaemia, diabetes mellitus, cerebrovascular disease, gall bladder disease, and some types of cancer. Almost all the Western anti-obesity drugs have adverse effects or body weight is regained upon cessation of therapy. Recent studies have found that acupuncture had a similar efficacy as the Western anti-obesity drugs with fewer reported adverse effects. However, these conclusions were limited due to the small sample size and low quality of methodologies of these studies. Therefore, we design this study to explore the effectiveness and safety of acupuncture on weight control.
\end{abstract}

Methods/design: This is a pilot single-blinded, randomized, sham-controlled trial on acupuncture for body weight control. Seventy-two participants are randomly assigned to the acupuncture group or the control group. Tianshu (ST-25), Daheng (SP-15), Daimai (GB-26), Qihai (CV-6), Zhongwan (CV-12), Zusanli (ST-36), Fenglong (ST-40), and Sanyinjiao (SP-6) are selected as acupuncture points. For the acupuncture group, disposable acupuncture needles will be inserted at a depth of 10-25 $\mathrm{mm}$ into the points and electrical stimulation with dense-disperse waves at $50 \mathrm{~Hz}$ and $10 \mathrm{~V}$ will be applied on the abdominal points. The bodily needles will be retained for 30 minutes. For subjects assigned to the control group, Streitberger's non-invasive acupuncture needles will be applied to serve as the sham control at the same acupoints with the same stimulation modality, except that the needles are only adhered to the skin instead of inserted. The duration of the treatment is 8 weeks with two sessions per week, and the follow-up period is 8 weeks. The primary outcome is the change in body weight before and after treatment. The secondary outcomes include changes in body mass index, waist circumference, hip circumference, and body fat percentage during the treatment and follow-up period.

Discussion: The study will compare the efficacy and safety of acupuncture with sham acupuncture on weight control, in the hope of obtaining evidence for utilizing acupuncture for body weight control.

Trial registration: NCT02516878. Registered on 30 July 2015.

\footnotetext{
* Correspondence: bzxiang@hkbu.edu.hk

${ }^{1}$ Hong Kong Chinese Medicine Study Centre, Hong Kong Baptist University,

AAB 105, Hong Kong Baptist University, Kowloon Tong, Kowloon, Hong Kong

${ }^{2} \mathrm{~S} c h \mathrm{ool}$ of Chinese Medicine, Hong Kong Baptist University, 3/F, Jockey Club

School of Chinese Medicine Building, 7 Baptist University Road, Kowloon

Tong, Kowloon, Hong Kong

Full list of author information is available at the end of the article
} 


\section{Background}

Obesity is an increasingly prevalent chronic condition that is associated with serious morbidity and mortality [1]. According to the statistical data of the Centre for Health Protection, Hong Kong SAR in 2013, 36.6 \% of the population aged 18-64 were classified as overweight or obese (body mass index, BMI $\geq 23$ ), including $18.8 \%$ as obese $(B M I \geq 25)$ among all the population. Excess body weight is the sixth most important risk factor contributing to the overall burden of disease worldwide, such as hypertension, heart disease, hypercholesterolaemia, diabetes mellitus, cerebrovascular disease, gall bladder disease, and some types of cancer [2, 3]. Obesity is becoming a global epidemic and common health problem. The weight control treatments commonly used nowadays include behavioural intervention, dietary intervention and physical activity, which require a higher level of self-discipline and take up to 6 months or longer to achieve significant weight change [4]; surgery like the duodenal-jejunal bypass liner, which may cause surgical complications [5]; and anti-obesity medications.

Currently, most of the Western anti-obesity drugs have adverse effects like oily discharge followed by flatus and faecal incontinence for orlistat [6]; headache, nausea and dizziness for lorcaserin [7] or body weight regaining upon cessation of therapy [8]. More people would like to seek help from Chinese herbal medicine (CHM) and/or acupuncture for body weight control, especially from acupuncture because it is safe and does not involve the intake of drugs [9].

Systematic reviews and randomized controlled trials have shown the beneficial effects of acupuncture, including body acupuncture and auricular acupressure $[9,10]$. Acupuncture compared with placebo or lifestyle modification had more effectiveness in lowering body weight, BMI and waist circumference and it had a similar efficacy as the Western anti-obesity drugs with fewer reported adverse effects of insomnia, headache or gastrointestinal reactions [10, 11]. Auricular acupressure has been summarized as having the effect of appetite suppression on overweight patients [12], and its efficacy on weight control is higher when combined with body acupuncture [13]

However, these conclusions are limited due to the small sample size and low quality of the methodologies of these studies. Also, there is no restricted research to investigate the combination effects of body acupuncture and auricular acupressure compared to sham acupuncture for body weight control among Hong Kong's Chinese population. Therefore, we design this single-blinded, randomized controlled clinical trial to explore the effectiveness, efficacy and safety of body acupuncture and auricular acupressure on weight control in Hong Kong.

\section{Objective}

The aim of the study is to assess the efficacy and safety of body acupuncture and auricular acupressure compared to sham acupuncture on body weight control through a pilot randomized controlled study.

\section{Methods/design \\ Study design}

This is a pilot single-blinded, randomized, sham-controlled trial on acupuncture for body weight control conducted at Hong Kong Baptist University Chinese Medicine Clinics. Seventy-two participants will be enrolled from the public through advertisements via the university's website and newspaper. Subjects will be randomly assigned to the acupuncture group or the control group. Treatment will be given with two sessions per week for 8 weeks, and the post-treatment follow-up period will be 8 weeks. The primary outcome is the change in body weight before and after treatment. The total study period will be 16 weeks. The study protocol has been approved by the Hong Kong Baptist University Ethics Committee on the Use of Human Subjects for Teaching and Research (Approval no. HASC/13-14/ 0266) and registered in ClinicalTrials.gov (NCT02516878). The flow chart of the trial is shown in Fig. 1. The checklist for items in STRICTA 2010 is given in Table 1. Informed consent is obtained from each participant.

\section{Participants \\ Setting}

The study is conducted in the research and clinical centres, School of Chinese Medicine, Hong Kong Baptist University.

\section{Inclusion criteria}

Patients who meet all of the following criteria will be eligible for the study: men or women aged between 18 and 65 years old; with body mass index $(\mathrm{BMI}) \geq 25 \mathrm{~kg} / \mathrm{m}^{2}$; who have not received any other weight control measures or any medical and/or drug history within the last 3 months.

\section{Exclusion criteria}

Patients who meet any of the following criteria will be excluded from the study: those with endocrine disease, for example, thyroid disorder, pituitary disorder or sex gland disorder; those with heart disease, for example, arrhythmia, heart failure, myocardial infarction or patients with pacemakers; those with allergy and immunology disease; those having a bleeding tendency; pregnant or lactating women; those having impaired hepatic or renal function; those who have had a stroke or are otherwise unable to exercise. 


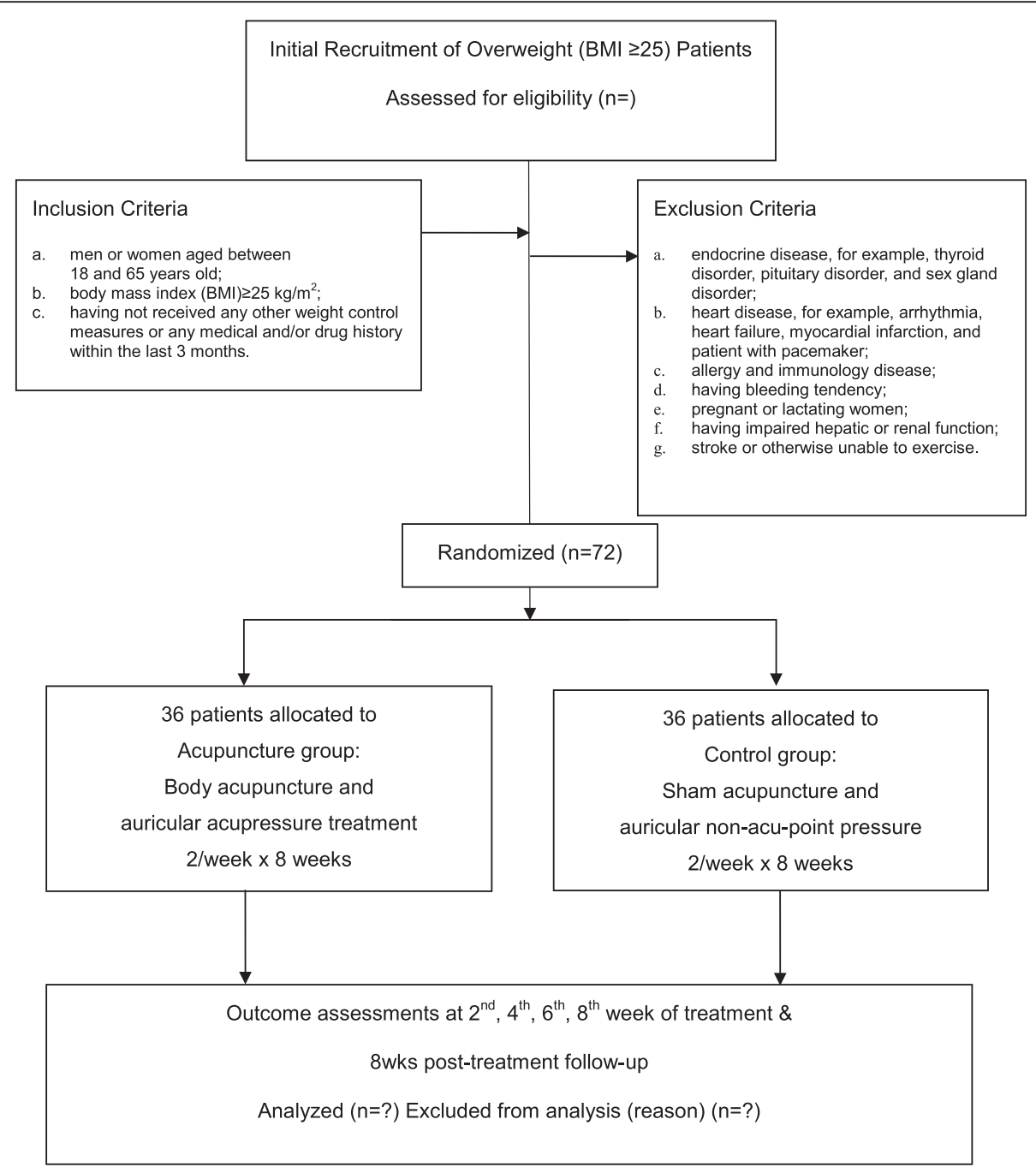

Fig. 1 Participant flow diagram

\section{Interventions}

\section{Body acupuncture treatment}

Acupuncture intervention will be conducted for two sessions per week over 8 consecutive weeks. According to a systematic review, the highly frequently used acupuncture points in body weight control trials are Zusanli (ST-36), Sanyinjiao (SP-6), Tianshu (ST-25), Fenglong (ST-40), Zhongwan (CV-12) and Qihai (CV-6) [9]. Among them, the traditional effects of the abdominal points are harmonizing gastrointestinal function, regulating Qi circulation and treating localized problems, which would be excessive adipose tissue deposition for the studied subjects. The traditional effects of the points at the lower limbs are stabilizing gastrointestinal function and enhancing fluid drainage. With the clinical experience of our principal investigator and co-investigators, eight body points are chosen: Tianshu (ST-25), Daheng (SP-15), Daimai (GB-26), Qihai (CV-6), Zhongwan (CV-12),
Zusanli (ST-36), Fenglong (ST-40) and Sanyinjiao (SP-6). The details of acupoints and their functions are listed in Table 2. The acupuncture treatment will be conducted by a registered Chinese medicine practitioner with more than 6 years of Chinese medicine college education and at least 5 years of clinical experience.

Disposable acupuncture needles (verum acupuncture needles asia-med Special No. $160.30 \times 30 \mathrm{~mm}$ matching the Streitberger sham needles) will be inserted at a depth of $10-25 \mathrm{~mm}$ into the points. As electro-acupuncture has been reviewed as having a higher efficiency in weight loss treatment [14], we will deliver electrical stimulation with dense-disperse waves at $50 \mathrm{~Hz}$ and $10 \mathrm{~V}$ through the electrical acupuncture stimulation instrument (ES-160 6-Channel Programmable Electro-acupuncture) to the abdominal points. The bodily needles will be retained for 30 minutes. 
Table 1 Checklist for items in STRICTA 2010*

\begin{tabular}{ll}
\hline Item & Detail \\
\hline $\begin{array}{l}\text { 1. Acupuncture } \\
\text { rationale }\end{array}$ & 1a) Style of acupuncture: \\
& According to systematic reviews and clinical \\
& experiences of our principal investigator \\
& and co-investigators. Manual and \\
& electro-acupuncture based on traditional \\
& Chinese medicine theory \\
& 1b) Reasoning for treatment provided, based \\
& on historical context, literature sources, and/or \\
& consensus methods, with references \\
& where appropriate
\end{tabular}

1c) Extent to which treatment was varied:

Standard treatment is used. No variation of treatment among patients

2. Details of needling

2a) Number of needle insertions per subject per session (mean and range where relevant):

14 needles

2b) Names (or location if no standard name) of points used (uni/bilateral)

Bilateral:

Tianshu (ST-25), Daheng (SP-15), Daimai (GB-26),

Zusanli (ST-36), Fenglong (ST-40),

Sanyinjiao (SP-6)

Unilateral:

Qihai (CV-6), Zhongwan (CV-12)

2c) Depth of insertion, based on a specified unit of measurement or on a particular tissue level:

10-25 mm

2d) Response sought (e.g. de qi or muscle twitch response):

De qi

2e) Needle stimulation (e.g. manual, electrical):

Manual and electrical - dense-disperse waves at $50 \mathrm{~Hz}$ and $10 \mathrm{~V}$

2f) Needle retention time:

$30 \mathrm{~min}$

2g) Needle type (diameter, length and manufacturer or material):

Disposable acupuncture needles (verum acupuncture needles asia-med Special No. 16 with $0.30 \times 0.30 \mathrm{~mm}$ matching the Streitberger sham needles)

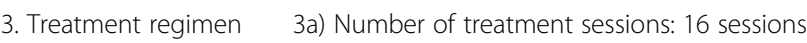

3b) Frequency and duration of treatment sessions:

2/week for 8 consecutive weeks

4. Other components of 4a) Details of other interventions administered treatment to the acupuncture group (e.g. moxibustion, cupping, herbs, exercises, lifestyle advice):

Auricular acupressure with Semen Vaccariae embedded adhesive tape.

Unilateral auricular points: Hunger, Shen men, Spleen and Stomach

Table 1 Checklist for items in STRICTA 2010* (Continued)

4b) Setting and context of treatment, including instructions to practitioners, and information and explanations to patients:

University Clinics

Participants will be informed about acupuncture treatment in the study as follows: "In this study, acupoints for weight control will be used based on related reports and clinical experience of our investigators."

5. Practitioner $\quad$ 5) Description of participating acupuncturists background (qualification or professional affiliation, years in acupuncture practice, other relevant experience):

Hong Kong registered Chinese medicine practitioners having at least 3 years of clinical experience, who have undergone training and are able to provide identical acupuncture treatment in accordance with a predefined protocol

6. Control or comparator interventions

6a) Rationale for the control or comparator in the context of the research question, with sources that justify this choice:

To assess the efficacy and safety of body acupuncture and auricular acupuncture compared to sham acupuncture

$6 b)$ Precise description of the control or comparator. If sham acupuncture or any other type of acupuncture-like control is used, provide details as for items 1 to 3 above

- Style of acupuncture:

Sham acupuncture + Semen Vaccariae embedded tape pressure on non-acupoints at auricular helix

Table 1 Click here to download Table Table 1.docx

- Number of needle insertions per subject per session:

14 sham needles at the same acupoints as the treatment group, and 4 auricular helix points with embedded tape pressure

- Depth of insertion:

Needles are only adhered to the skin.

- Needle retention time:

$30 \min$

- Needle type

Streitberger's non-invasive acupuncture needles (Gauge $8 \times 1.2^{\prime \prime} / 0.30 \times 30 \mathrm{~mm}$ )

- Number of treatment sessions:

16 sessions

- Frequency and duration of treatment sessions:

2/week for 8 consecutive weeks

*This checklist, which should be read in conjunction with the explanations of the STRICTA items, is designed to replace CONSORT 2010's item 5 when reporting an acupuncture trial 
Table 2 Acupoints of the body and their functions

\begin{tabular}{|c|c|}
\hline Acupoint & Classical effects of stimulation \\
\hline Tianshu (ST-25) & $\begin{array}{l}\text { Restoring and harmonizing the flow of energy in } \\
\text { the intestines; regulating the Qi; breaking up blocks }\end{array}$ \\
\hline Daheng (SP-15) & $\begin{array}{l}\text { Expelling cold in digestive system; regulating } \\
\text { gastrointestinal functions }\end{array}$ \\
\hline Daimai (GB-26) & $\begin{array}{l}\text { Directing the Qi down into the lower body; } \\
\text { stabilizing and harmonizing the lower tri-energizer } \\
\text { (lower Jiao), thus draining dampness }\end{array}$ \\
\hline Qihai (CV-6) & $\begin{array}{l}\text { Supplementing and regulating Qi and Yang; } \\
\text { stabilizing and nourishing the kidneys }\end{array}$ \\
\hline Zhongwan (CV-12) & $\begin{array}{l}\text { Regulating the stomach meridian, harmonizing } \\
\text { stomach Qi; draining fluids }\end{array}$ \\
\hline Zusanli (ST-36) & $\begin{array}{l}\text { Stabilizing and regulating the stomach meridian, } \\
\text { harmonizing Qi and the blood }\end{array}$ \\
\hline Fenglong (ST-40) & Transforming body fluids; expelling phlegm \\
\hline Sanyinjiao (SP-6) & $\begin{array}{l}\text { Regulating the spleen, liver and kidney meridian; } \\
\text { intensifying and dynamizing fluids circulation }\end{array}$ \\
\hline
\end{tabular}

\section{Auricular acupressure}

Participants of the treatment group will additionally receive unilateral auricular acupressure at four auricular points as Hunger, Shen men, Spleen and Stomach with Semen Vaccariae (Wang Bu Liu Xing) embedded within adhesive tape at each treatment session (Fig. 2). Acupressure will be self-applied by the subjects with repeated pressing of the tape with fingertips for 1 minute per point, thrice per day. The embedded tape will be retained in situ for 24 hours, and then the alternate ear will be treated at the next visit. The four points were most frequently used on overweight treatment as summarized by a systemic review [9]. Clinically, the ear points Spleen and Stomach are used for regulating gastrointestinal function; Shen men, meaning Spirit gate, is used for calming mental conditions; Hunger is used for suppressing the desire to eat and is reported to be particularly effective on weight loss [15].

\section{Sham acupuncture}

For subjects assigned to the control group, Streitberger's non-invasive acupuncture needles (Gauge $8 \times 1.2$ "/ $/ 0.30 \times$ $30 \mathrm{~mm}$ ) will be applied to serve as a sham control at the same acupoints with the same stimulation modality, except that the needles are only adhered to the skin instead of being inserted [16]. The validity and credibility of this system has been well demonstrated $[17,18]$.

\section{Sham auricular acupressure}

The Semen Vaccariae embedded tape used in the treatment group will be applied on four non-acupoints at the helix unilaterally and retained for 24 hours, and then the alternate ear will be used at the next visit (Fig. 2). The helix region was suggested to be used as a control in

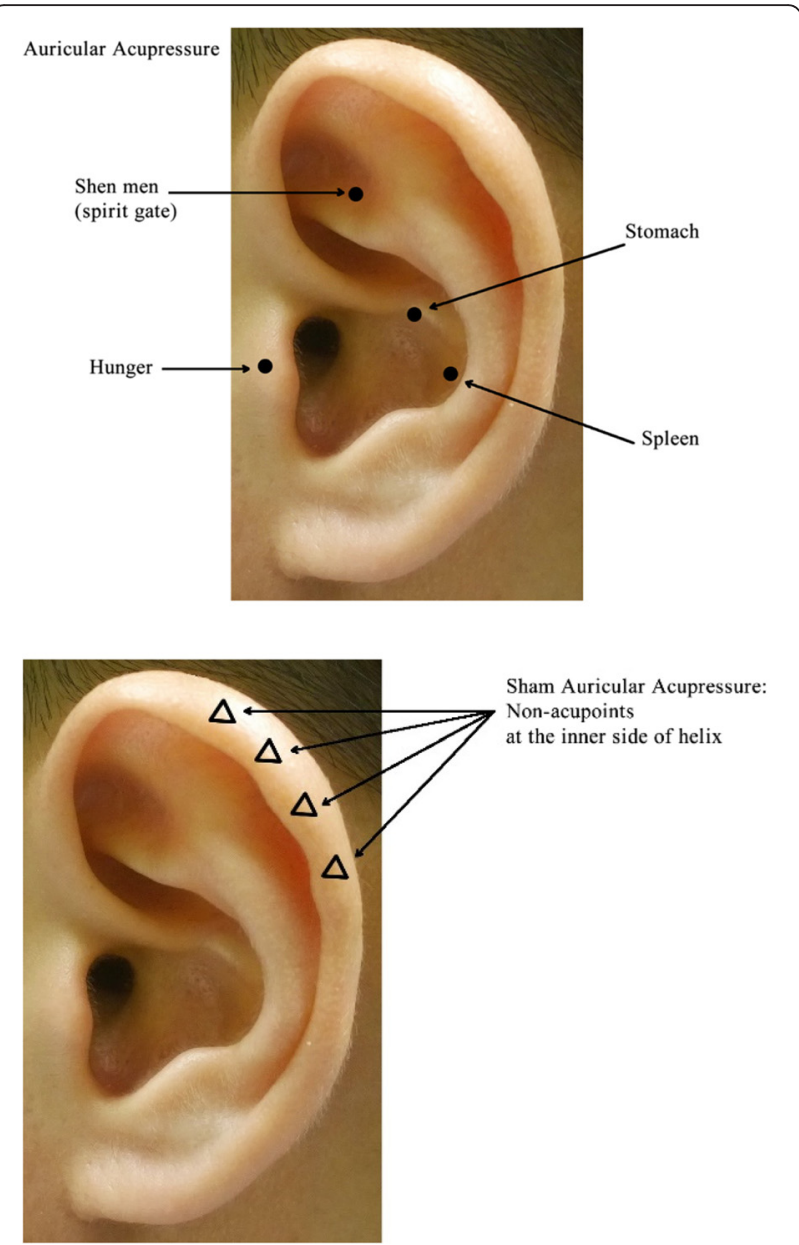

Fig. 2 Auricular acupressure points and sham acupressure points

auricular needling and was applied in previous clinical trials $[19,20]$.

All subjects will be advised to have a regular number of meals daily and not to have any snacks. Meals comprise one bowl of rice $(210 \mathrm{~g})$ for subjects $>70 \mathrm{~kg}$ and two-thirds of a bowl of rice $(140 \mathrm{~g})$ for those $<70 \mathrm{~kg}$, with instructions to eat side dishes balanced with the rice. Also, subjects will be instructed not to perform any exercise other than that required for their daily work.

\section{Outcome measures}

The primary outcome is the change in body weight before and after treatment. The secondary outcomes include changes in BMI, waist circumference, hip circumference and body fat percentage during the treatment and followup period. Besides at baseline ( 0 week), the numbers will be measured every 2 weeks. Both the body weight and body fat percentage will be measured with the Omron Karada Scan HBF-701.

Adverse events will be noted throughout the study, based on participant reports and laboratory tests (whole 
blood counts, renal and liver functions) if needed. All clinical adverse events will be recorded according to terms of intensity (mild, moderate or severe), duration, outcome and relationship to the study.

\section{Randomization assignment}

Subjects of both groups will be randomly assigned to receive acupuncture (body and auricular acupressure) or control (sham) treatment. For randomization, simple, complete non-sequential random numbers will be generated in advance by a computer program in a block of four, and kept by the principal investigator (PI, ZXB). After a patient's eligibility is confirmed, a randomization number which corresponds to the group allocation will be provided to the acupuncturist by the PI. This arrangement will ensure that the clinical assessor and participants are blinded to the allocation.

\section{Sample size}

The sample size was calculated based on the primary endpoint of changes in body weight. A systematic review on acupuncture for obesity has shown that acupuncture significantly reduced the body weight with average weight loss $(\mathrm{MD}=1.56 \mathrm{~kg}, 95 \% \mathrm{CI}=0.74-$ 2.38) [6]. Therefore, a sample size of 60 should be provided to achieve a significance level of $\alpha=0.05$ with a power $(1-\beta)$ of $90 \%$ using a two-sample $t$ test. The number of subjects increases to 72 when estimated $20 \%$ dropouts are considered.

\section{Data processing and analysis}

All efficacy and safety analyses will be conducted according to the intention-to-treat (ITT) principle. Missing values will be imputed by the last-observationcarried-forward method. The statistical analysis will be performed using the Statistical Package for the Social Sciences (SPSS) for Windows version 21.0. The statistical significance is defined as two-sided $P$ value of $<0.05$. Baseline characteristics will be reported as mean (SD). Baseline differences between the groups will be assessed with the use of Student's $t$ test for normally distributed continuous variables and the non-parametric Mann-Whitney $U$ test for non-normally distributed variables. For categorical variables, the chi-squared test or Fisher's exact test will be used. Comparisons between groups will be conducted by using an analysis of covariance (ANCOVA) with baseline as covariate. All items and subscales will be compared between groups every 4 weeks using ANCOVA, with treatment group as a factor in the model and baseline as the covariate. The changes from baseline to endpoint of treatment in scores will be tested with a repeated measures analysis of variance (ANOVA). Within-group differences will be assessed with a paired $t$ test for normally distributed data and a Wilcoxon signed-rank test for non-normally distributed data.

\section{Discussion}

This single-blinded, randomized controlled clinical trial aims to evaluate the efficacy and safety of acupuncture on body weight control in Hong Kong. It will be the first such study on the Hong Kong population and will obtain evidence for utilizing acupuncture in obesity treatment especially for patients with Hong Kong diet patterns and health conditions. In order to achieve a higher therapeutic efficacy, we will integrate electro-acupuncture with auricular acupressure. Except for acupuncture point stimulation on the body and ears, we will not apply any interventions such as CHM, diet or physical activities to the subjects. Therefore, the effects of acupuncture alone will be observed. The outcome measures will include the change in body weight and body fat percentage, which will provide data to us on analysis of the treatment's potential benefit on body fat mass.

So far there is no study of this kind in Hong Kong. This pilot study will provide evidence for large-scaled research such as combined therapy on overweight subjects with CHM, diet control, physical training or behavioural intervention. Further research on the changes in hormones, the nervous system or psychological conditions can also be developed to specifically target the Hong Kong population, as there have only been overseas studies up to the present.

In this clinical trial, the selection of body and auricular points is standardized and utilized for every subject. This may be helpful for easier utilization of the treatment over different individuals, but the limitation is that the selection of acupuncture points is not based on syndrome differentiation, which is the main concern in traditional Chinese acupuncture. Another limitation is the small sample size, as the trial was proposed to be a pilot study for a later large-scaled clinical trial.

In conclusion, in this pilot study, a single-blinded, randomized controlled clinical trial will be conducted to evaluate the effectiveness, efficacy and safety of acupuncture on weight control in Hong Kong. This study will obtain the solid evidence for Chinese medicine practitioners (CMPs) to utilize acupuncture for obesity and will also provide a platform to offer research training opportunities for junior CMPs.

\section{Trial status}

The participants are currently being recruited for the present study.

\section{Abbreviations}

ANCOVA, analysis of covariance; ANOVA, analysis of variance; BMI, body mass index; CHM, Chinese herbal medicine; CMP, Chinese medicine practitioner;

$\mathrm{ITT}$, intention-to-treat 


\section{Acknowledgements}

This research was financially supported by Hong Kong Hospital Authority Research Project: Acupuncture for Body Weight Control: A Pilot Randomized Controlled Trial (Ref Code: HA 33-14-112-542-130).

Written informed consent was obtained from the patient(s) for publication of this manuscript and accompanying images. A copy of the written consent is available for review by the Editor-in-Chief of this journal.

\section{Authors' contributions}

ZXB is the corresponding author and also designed the study. LLDZ drafted the manuscript. WK and TFL enroll the participants. SPZ, JJY and TFL revised the manuscript. TCZ and BN provided critical advice. All authors read and approved the final manuscript.

\section{Competing interests}

The authors declare that they have no competing interests.

\section{Author details}

'Hong Kong Chinese Medicine Study Centre, Hong Kong Baptist University, AAB 105, Hong Kong Baptist University, Kowloon Tong, Kowloon, Hong Kong. ${ }^{2}$ School of Chinese Medicine, Hong Kong Baptist University, 3/F, Jockey Club School of Chinese Medicine Building, 7 Baptist University Road, Kowloon Tong, Kowloon, Hong Kong. ${ }^{3}$ Chinese Medicine Department, Hong Kong Hospital Authority, Kowloon, Hong Kong.

Received: 11 January 2016 Accepted: 29 June 2016

Published online: 25 July 2016

\section{References}

1. Lehnert T, et al. Health burden and costs of obesity and overweight in Germany: an update. Eur J Health Econ. 2015;16(9):957-67.

2. Carnethon MR. Diabetes mellitus in the absence of obesity: a risky condition. Circulation. 2014;130(24):2131-2.

3. Alexandre L, Long E, Beales IL. Pathophysiological mechanisms linking obesity and esophageal adenocarcinoma. World J Gastrointest Pathophysiol. 2014:5(4):534-49.

4. Dombrowski SU, et al. Behavioural interventions for obese adults with additional risk factors for morbidity: systematic review of effects on behaviour, weight and disease risk factors. Obes Facts. 2010;3(6):377-96.

5. Ingrid $Z$, et al. The duodenal-jejunal bypass liner for the treatment of type 2 diabetes mellitus and/or obesity: a systematic review. Obes Surg. 2014;24:310-23.

6. Viner RM, et al. Efficacy and safety of anti-obesity drugs in children and adolescents: systematic review and meta-analysis. Obes Rev. 2010;11(8):593-602

7. Chan EW, et al. Efficacy and safety of lorcaserin in obese adults: a metaanalysis of 1-year randomized controlled trials (RCTs) and narrative review on short-term RCTs. Obes Rev. 2013;14(5):383-92.

8. Scheen AJ, Van Gaal LF. Combating the dual burden: therapeutic targeting of common pathways in obesity and type 2 diabetes. Lancet Diabetes Endocrinol. 2014;2(11):911-22.

9. Sui $Y$, et al. A systematic review on use of Chinese medicine and acupuncture for treatment of obesity. Obes Rev. 2012;13(5):409-30.

10. Cho SH, et al. Acupuncture for obesity: a systematic review and metaanalysis. Int J Obes (Lond). 2009;33(2):183-96.

11. Lin XM, et al. Systematic evaluation of therapeutic effect of acupuncture for treatment of simple obesity. Zhongguo Zhen Jiu. 2009;29(10):856-60.

12. Lacey JM, et al. Acupuncture for the treatment of obesity: a review of the evidence. Int J Obes. 2003;27:419-27.

13. Wei QL, et al. Treatment of simple obesity with auricular acupuncture, body acupuncture and combination of auricular and body acupuncture. Chin J Clin Rehabil. 2004;8(21):4357-9.

14. Belivani $M$, et al. Acupuncture in the treatment of obesity: a narrative review of the literature. Acupunct Med. 2013;31:88-97.

15. Yeo $S$, et al. Randomised clinical trial of five ear acupuncture points for the treatment of overweight people. Acupunct Med. 2014;32:132-38.

16. Shin KM, et al. Acupuncture for lateral epicondylitis (tennis elbow): study protocol for a randomized, practitioner-assessor blinded, controlled pilot clinical trial. Trials. 2013;14:174.

17. Fleckenstein J, et al. Acupuncture for post anaesthetic recovery and postoperative pain: study protocol for a randomised controlled trial. Trials. 2014;15:292
18. Man SC, et al. A pilot controlled trial of a combination of dense cranial electroacupuncture stimulation and body acupuncture for post-stroke depression. BMC Complement Altern Med. 2014;14:255.

19. Margolin A, et al. A single-blind investigation of four auricular needle puncture configurations. Am J Chin Med. 1995;23(2):105-14.

20. Usichenko $\mathrm{Tl}$, et al. Auricular acupuncture for pain relief after total hip arthroplasty - a randomized controlled study. Pain. 2005;114:320-27.

\section{Submit your next manuscript to BioMed Central and we will help you at every step:}

- We accept pre-submission inquiries

- Our selector tool helps you to find the most relevant journal

- We provide round the clock customer support

- Convenient online submission

- Thorough peer review

- Inclusion in PubMed and all major indexing services

- Maximum visibility for your research

Submit your manuscript at www.biomedcentral.com/submit 